\title{
Panorama da primeira década pós-promulgação do Estatuto do Idoso
}

\author{
Anelise Crippa*, Carla Helena Augustin Schwanke*"
}

\section{Resumo}

Este trabalho apresenta uma revisão sistemática a respeito do Estatuto do Idoso (EI) lei n. 10.741/2003 que regulamenta os direitos dos idosos residentes no território brasileiro. Foram identificados 23 artigos, dos quais 21 foram, efetivamente, incluídos na revisão. Tais artigos abordam o conhecimento dos idosos, frequentadores de universidade aberta da terceira idade, acerca da cidadania e do envelhecimento (um artigo); a percepção de profissionais da saúde que atuam em hospital geriátrico em relação às mudanças ocorridas na assistência ao idoso após a promulgação do El (um artigo); a violência sob diferentes perspectivas; tipos de violência, perfil do agredido e do agressor, obrigatoriedade de notificação de violência pelo profissional da saúde (seis artigos); a educação tanto do aspecto de melhoria de relação e respeito das outras gerações com o idoso, quanto direito dos idosos à educação (três artigos); um panorama legislativo referente aos direitos do idoso (dois artigos); as políticas públicas existentes no Brasil referente ao idoso (quatro artigos); os direitos estabelecidos no Estatuto do Idoso em relação à saúde bucal (um artigo); a inserção do nutricionista no El (um artigo); o relato da experiência de trabalhar atividades de lazer e saber com um grupo de idosos da Estratégia Saúde da Família (um artigo); e a análise do conteúdo do El, identificando imagens e sentidos básicos atribuídos à velhice (um artigo). Dessa forma, observou-se que o assunto mais frequente nas publicações científicas é a violência contra os idosos, constatado em $29 \%$ das publicações sobre o El.

Palavras-chave: Envelhecimento. Idosos. Legislação. Publicações.

* Advogada, Especialista em Direito de Família e em Direito Processual Civil. Mestre em Gerontologia Biomédica. Pesquisadora do Laboratório de Bioética e de Ética Aplicada a Animais do Instituto de Bioética da Pontifícia Universidade Católica do Rio Grande do Sul (PUCRS). Endereço para correspondência: Av. Ipiranga, 6681 - Prédio 81 - 7ํandar, sala 703 - CEP: 90619-900 - Porto Alegre (RS), CEP: 90619-900. E-mail: anecrippa@gmail.com.

** Médica Geriatra, Doutora em Gerontologia Biomédica, Professora do Programa de Pós-Graduação em Gerontologia Biomédica do Instituto de Geriatria e Gerontologia da Pontifícia Universidade Católica do Rio Grande do Sul (PUCRS). Endereço para correspondência: Avenida Ipiranga, n. 6690, 3º andar, Porto Alegre (RS), CEP: 90610-000. E-mail: schwanke@pucrs.br.

$\rightarrow$ http://dx.doi.org/10.5335/rbceh.2013.3143 


\section{0 envelhecimento populacional no Brasil}

O incremento do número da população idosa em nosso país e as peculiaridades que permeiam as suas necessidades apresenta-se como um cenário cada vez mais presente. Relatos longínquos já norteavam essa ceara, expressando preocupações quanto aos cuidados, ao relacionamento familiar e às questões éticas do envelhecimento. Nesse sentido, pode-se citar a obra Saber Envelhecer, de Cícero (2010), datada de 44 a.C., bem como o livro Rei Lear, de Shakespeare (2007), escrito em 1606.

O crescimento populacional, em números absolutos e relativos, é um fenômeno mundial sem precedentes. Em 1950, tínhamos, no mundo, um total de 204 milhões de idosos. Em 1998, quase cinco décadas depois, esse número passou para 579 milhões, ou seja, houve um crescimento de quase 8 milhões de pessoas idosas por ano. As projeções são de um total de 1.900 milhões de pessoas idosas, para 2050 (IBGE, 2010).

No Brasil, o censo demográfico 2010 do Instituto Brasileiro de Geografia e Estatística (IBGE), cujos dados foram divulgados em 2011, mostram um aumento da expectativa de vida dos brasileiros e, por sua vez, um crescimento do número de idosos. Estima-se que, em 2050, a população idosa represente $18 \%$ do total de brasileiros, o que corresponderá, aproximadamente, a 47 milhões de idosos (IBGE, 2010).

O idoso é, presumivelmente, vulnerável por encontrar-se, especialmente exposto a riscos, devido à sua situação de fragilidade, como, por exemplo, a ocor- rência de doenças crônicas, associadas às hospitalizações e ao repouso prolongado no leito, fatos que acabam potencializando suas condições de vulnerabilidade (LOCH et al., 2012).

O Estado tem importante papel de cuidador e protetor do idoso, possibilitando, com as políticas públicas, a garantia das "oportunidades e facilidades para preservação de sua saúde física e mental e seu aperfeiçoamento moral, intelectual, espiritual e social" (PONTES, 2012). Assim, é dever do Estado combater as situações que possibilitem desencadeamento da vulnerabilidade, por meio do Direito que estabelece o conjunto de normas jurídicas (PERES, 2009).

\section{Um panorama sobre o Estatuto do Idoso}

Instrumento ímpar para a proteção dos idosos, foi promulgada, em 2003, a lei n. 10.741 denominada Estatuto do Idoso (EI). Dividido em sete títulos e, por sua vez, alguns são subdivididos em capítulos, e compostos de 118 artigos.

O Estatuto do Idoso é uma compilação de normativas já existentes, bem como de novos dispositivos legais, baseando-se no Estatuto da Criança e do Adolescente (ECA), o qual discorre sobre cada um dos direitos e especifica as punições para os infratores, possibilitando, com isso, uma facilitação da sua compreensão e aplicação (MARTINEZ, 2012).

Como cerne dos artigos, encontra-se o amparo, a assistência e a proteção ao indivíduo idoso. O EI elucida os deveres da família, das instituições, do cidadão e do Estado em relação aos cuidados e ao apoio ao idoso (BRASIL, 2002). 
Embora o marco escolhido para caracterização do idoso, pelo EI, seja o cronológico, são consideradas idosas as pessoas com idade igual ou superior a 60 anos. É importante enfatizar que alguns artigos são para os com idade igual ou superior a 65 anos, como a gratuidade no transporte coletivo e o benefício da prestação continuada (BEZERRA, 2012).

Diante de um instrumento tão importante e garantidor, é fundamental que se identifiquem os estudos elaborados a partir do Estatuto do Idoso.

Revisão da literatura sobre o Estatuto do Idoso

A presente investigação teve por objetivo identificar e descrever os artigos publicados na base de dados Scientific Electronic Library Online (Scielo), Publicações Médicas (Pubmed), Literatura Latino-Americana e do Caribe em Ciências da Saúde (Lilacs) e Índice Bibliográfico Espanhol de Ciências de Saúde (Ibecs), com a palavra-chave Estatuto do Idoso, desde 2003, ano da sua promulgação, resultados apresentados na Tabela 1.
Tabela 1 - Artigos científicos localizados nas bases de dados Lilacs, Ibecs, Pubmed e Scielo com a palavra-chave Estatuto do Idoso

\begin{tabular}{lc}
\hline \multicolumn{1}{c}{ Base de dados } & Números de artigos \\
\hline Lilacs & 21 \\
Ibecs & 1 \\
Pubmed & 1 \\
Scielo & 8 \\
\hline
\end{tabular}

Fonte: elaborada pelas autoras com base em dados de dezembro de 2012.

Os artigos identificados nas bases Ibecs e Pubmed aparecem repetidamente na base de dados Lilacs, de forma igual com seis dos oito artigos da base de dados Scielo. Assim, elegeu-se apresentar os artigos encontrados na base de dados Lilacs, por essa ser a mais completa (Quadro1). Na sequência, apresenta-se uma descrição de cada publicação. 
Quadro 1 - Artigos científicos localizados nas bases de dados Lilacs com a palavra-chave Estatuto do Idoso

\begin{tabular}{|c|c|c|}
\hline Autores & Ano & Título do artigo \\
\hline Gianiselle et al. & 2004 & Estatuto do Idoso: aspectos jurídicos, sociais e culturais \\
\hline Pereira et al. & 2005 & Políticas sobre envelhecimento e saúde no mundo \\
\hline Miyata et al. & 2005 & $\begin{array}{l}\text { Políticas e programas na atenção à saúde do idoso: um } \\
\text { panorama nacional }\end{array}$ \\
\hline Camargos et al. & 2006 & $\begin{array}{l}\text { Política, estado e sociedade: o estatuto do idoso e a aten- } \\
\text { ção à saúde }\end{array}$ \\
\hline Laks J. et al. & 2006 & $\begin{array}{l}\text { Psiquiatria forense e direitos humanos nos pólos da vida: } \\
\text { crianças, adolescentes e idosos }\end{array}$ \\
\hline Hebling E. et al. & 2006 & O Estatuto do Idoso e a saúde bucal \\
\hline Saliba O. et al. & 2007 & $\begin{array}{l}\text { Responsabilidade do profissional de saúde sobre a notifica- } \\
\text { ção de casos de violência doméstica }\end{array}$ \\
\hline Cavalcante PMT et al. & 2007 & $\begin{array}{l}\text { Em busca de um viver saudável: relato de experiência com } \\
\text { um grupo de idosos cadastrados na estratégia saúde da } \\
\text { família de Pacatuba - CE }\end{array}$ \\
\hline Martins MS. et al. & 2008 & $\begin{array}{l}\text { Mudanças na assistência ao idoso após promulgação do Es- } \\
\text { tatuto do Idoso segundo profissionais de hospital geriátrico }\end{array}$ \\
\hline Gomes L. et al. & 2008 & Violência contra o ancião \\
\hline Silva ACAP & 2008 & $\begin{array}{l}\text { Conhecimento, cidadania e direito do idoso: relatos pós-lei } \\
\text { n. } 10.741 / 2003\end{array}$ \\
\hline Camargos CN & 2009 & $\begin{array}{l}\text { Panorama de interiores: o profissional nutricionista e o Es- } \\
\text { tatuto do Idoso }\end{array}$ \\
\hline Moimaz SAS et al. & 2009 & $\begin{array}{l}\text { O idoso no Brasil: aspectos legislativos de relevância para } \\
\text { profissionais de saúde }\end{array}$ \\
\hline Whitaker DCA & 2010 & $\begin{array}{l}\text { O idoso na contemporaneidade: a necessidade de se edu- } \\
\text { car a sociedade para as exigências desse novo ator social, } \\
\text { titular de direitos }\end{array}$ \\
\hline Justo JS et al. & 2010 & A velhice no Estatuto do Idoso \\
\hline Correa MR et al. & 2010 & $\begin{array}{l}\text { Políticas públicas: a construção de imagens e sentidos para } \\
\text { o envelhecimento humano }\end{array}$ \\
\hline Scortegagna PA et al. & 2010 & $\begin{array}{l}\text { Educação: integração, inserção e reconhecimento social } \\
\text { para o idoso }\end{array}$ \\
\hline Massi G et al. & 2010 & Práticas de letramento no processo de envelhecimento \\
\hline Souza ER et al. & 2010 & $\begin{array}{l}\text { Inserção do tema violência contra a pessoa idosa nas polí- } \\
\text { ticas públicas de atenção à saúde no Brasil }\end{array}$ \\
\hline Cavalcanti MLT et al. & 2010 & $\begin{array}{l}\text { Percepções de gestores e profissionais de saúde sobre a } \\
\text { atenção aos idosos vítimas de violências no município do } \\
\text { Rio de Janeiro-Brasil }\end{array}$ \\
\hline Pinheiro JS et al. & 2011 & $\begin{array}{l}\text { Perfil dos idosos que sofreram violência atendidos em uma } \\
\text { instituição de Salvador no ano de } 2008\end{array}$ \\
\hline
\end{tabular}

Fonte: elaborado pelas autoras, 2013. 
De acordo com Gianiselle et al. (2004), logo após o início da vigência do EI, surgiram três leituras do Estatuto do Idoso: a legal, a de pessoas comuns e a da mídia impressa. Inicialmente, é feita uma apresentação do seminário de um grupo de pesquisa que originou este artigo. Indagou-se, no grupo, o porquê do EI, debatendo-se os aspectos legais. Após, questionou-se o que os idosos sabiam a respeito, momento em que se entrevistaram alguns idosos. Foram referenciadas pelos idosos as informações que apareceram na mídia. Assim, em relação à mídia são mostradas reportagens abordando o EI.

Pereira et al. (2005) fizeram uma revisão bibliográfica e legislativa, identificando as principais políticas e programas sanitários empreendidos no mundo, direcionado aos idosos. Relatou-se experiência de alguns países, especificamente do Brasil, que teve um crescimento legislativo no que se refere ao idoso, após a Constituição Federal de 1988. Concluíram que, apesar de haver diversas leis nacionais e internacionais, os idosos ainda ficam excluídos de inúmeras ações governamentais.

De acordo com Miyata et al. (2005) as políticas, estratégias e ações governamentais são identificadas para a saúde do idoso, por meio de revisão de literatura. Os autores apresentam, no período analisado, que foram implementadas campanhas de vacinação pelo governo federal, programa de valorização e saúde do idoso, mutirão de cirurgia de cataratas e distribuição de medicamentos. Em termos estaduais, apenas Minas Gerais integralizou as ações previstas na Políti- ca Nacional de Saúde do Idoso. O estado de São Paulo destaca-se na aplicação das referidas ações.

Camargos et al. (2006) buscaram compreender a necessidade de inclusão oficial do profissional da saúde no que tange às políticas anunciadas no EI. Para tanto, efetuaram uma revisão literária e análise das legislações. Concluíram que a legislação tem uma função de reaproximar a população de idosos, impedindo que haja um abatimento do indivíduo, em decorrência do envelhecimento.

Uma revisão crítica é realizada por Laks et al. (2006), relacionando os atos de violência, a fragilidade e a dependência. Abordam, além do tema violência, a capacidade civil e a responsabilidade formal comparativa às crianças e aos adolescentes. Os autores apresentaram como forma de violência o abuso e os maus tratos, visto que o agressor é alguém em condição de superioridade. Acredita-se, também, haver falha do cuidador por não proteger nem prestar os cuidados básicos ao idoso. Como principais formas de violência são apontados os maus tratos físicos e psicológicos, a negligência e a exploração material. Ressalta-se ainda a importância que o profissional da saúde deve dar aos indícios de violência, já que nem sempre os idosos estão dispostos a denunciar. Os autores apresentaram o protótipo do idoso vitimizado como aqueles que têm "comprometimento cognitivo, isolado socialmente, vivendo com pessoas com problemas mentais, especialmente alcoolismo e depressão (2006, p. 83)". Para esses, o conhecimento do EI é 
fundamental, pois, dessa maneira os profissionais de saúde podem tomar as devidas providências, protegendo o idoso e fazendo com que sejam efetivas as leis destinadas a eles.

Os direitos estabelecidos no Estatuto do Idoso em relação à saúde bucal são descritos por Hebling et al. (2006). Esses identificaram a saúde bucal inserida no art. 15, o qual assegura a atenção integral à saúde do idoso. No mesmo dispositivo, no parágrafo 2 , inciso $\mathrm{V}, \mathrm{o}$ poder público é incumbido pelo fornecimento de medicamentos e próteses. Os autores ressaltam tanbém que o cirurgião-dentista que atender idosos deverá agir como um agente de saúde, uma vez que as doenças decorrentes do envelhecimento são fatores de risco para o desencadeamento de doenças periodontais, por isso, enfatiza-se a necessidade de inclusão dessas doenças nos centros de referência da rede de assistência pública. Como conclusão, os autores destacam a necessidade do cirurgião-dentista ter conhecimento do EI e das ações públicas adequarem-se a essa legislação.

Saliba et al. (2007) averiguaram a responsabilidade do profissional da saúde, em notificar os casos de violência, por meio de uma busca nas legislações brasileiras, e suas implicações legais, as quais foram analisadas, juntamente com os códigos de ética da medicina, da enfermagem, da odontologia e da psicologia. Com isso, pode-se concluir que há o dever da notificação, nos casos de conhecimento de violência contra a pessoa idosa, existindo penalidades em todos os códigos de ética analisados.
No caso de Cavalcante et al. (2010), foi relatado o trabalho com um grupo de idosos cadastrados na Estratégia Saúde da Família de Pacatuba- CE. Em um grupo de 28 idosos foi realizado atividades de lazer e saber, no qual estes escolhiam a temática das palestras, com duração de doze meses de atividade, firmando uma combinação com os idosos para aderirem ao tratamento medicamentoso. O objetivo foi afastar os idosos do isolamento social, ocupando o tempo ocioso. Pode-se concluir, então, que ocorreu um favorecimento à adesão ao tratamento, pois criou-se um vínculo entre a unidade e a família, os idosos adquiram conhecimento com as palestras que focaram $o$ processo de envelhecimento e a saúde.

A fim de verificar qual o contato da equipe multiprofissional com o EI e conhecer a percepção desta equipe na interferência do EI na assistência prestada, Martins et al. (2008) realizaram uma pesquisa, em um hospital geriátrico, com equipe multiprofissional, constituída por 35 profissionais. Desses, 21 participantes já haviam lido o EI, 13 leram parcialmente e um não leu. Dos entrevistados, 24 acreditam que ocorreram mudanças após a promulgação do EI, nove pensam que não ocorreram e dois não consideraram o assunto. Foi referido que as mudanças ocorreram entre os próprios profissionais e na forma como a assistência passou a ser prestada, relatando modificações institucionais, nas atitudes e na conscientização dos profissionais, dos idosos e de seus familiares. Já Gomes et al. (2008) apresentaram um panorama da violência no Brasil como a causa de potencialização 
da vulnerabilidade do idoso eressaltam que o ancião não deve ser isolado em sua família, a qual é considerada lugar de excelência, embora se saiba que grande parte da violência ocorre por parte dos familiares. O que deve ocorrer, segundo os autores é um gerenciamento das relações, conscientizando a família. Para esses, o primeiro passo para proteger os idosos da violência é aumentar o nível de conhecimento dos médicos e demais profissionais da saúde, uma vez que estes devem estar atentos aos indícios de maus tratos.

Silva (2008) investigou o conhecimento do idoso, de forma qualitativa, por meio de questões que envolvem o processo de envelhecimento, a atuação do Poder Judiciário nesse processo e a discriminação. Os sujeitos envolvidos na pesquisa eram idosos que participam de uma universidade aberta para terceira idade do Rio de Janeiro, a qual voltou-se à compreensão do significado de cidadania que os idosos têm, como a vivenciam e como a praticam. Foram entrevistados 14 idosos, os quais não reconheciam suas necessidades e demandas como questões legais, mas, sim, como naturais do envelhecimento, mostrando, dessa forma, um distanciamento entre os direitos concebidos e os direitos utilizados pelos idosos.

Outra pontuação objetivou discutir a inserção do profissional de nutrição no EI. Camargos (2009) utilizou, para tanto, publicações científicas, o próprio EI e o código de ética desses profissionais. Para o autor, o EI poderia ter incluído de forma mais adequada esse profissional, o qual está incluso, apenas, ao se referirem ao processo multidisciplinar de atenção à pessoa idosa. Nesse contexto, pensa-se que não se pode visualizar o idoso isolado e que se deve lidar com a alimentação como forma de possibilitar qualidade em sua longevidade.

Moimaz et al. (2009) apresentaram um panorama legislativo no Brasil relacionado à saúde. Foi possível perceber o considerável aumento normativo do direito dos idosos após a Constituição Federal de 1988, concluindo que o Estatuto do Idoso é a lei que apresenta maior heterogeneidade de situações protetivas a essa população.

De acordo com Whitaker (2010), o processo de envelhecimento, as mudanças sociais, e a alteração do papel do idoso na sua própria família o situa na posição de aposentado, em oposição a chefe da família. Para o autor, são necessárias medidas para alterar essa visão da sociedade, as quais resgatem a dignidade do idoso, a partir da educação. Assevera que, sobretudo os professores, as crianças e os adolescentes devem valorizar os idosos, reconhecendo-os como titulares de direitos.

O conteúdo do Estatuto do Idoso foi analisado por Justo et al. (2010) para identificar imagens e sentidos básicos atribuídos à velhice. Evidenciou-se ainda a tentativa de diferenciar o idoso como cidadão de direitos, marcado por características próprias e que requer proteção e cuidado. Porém, ao analisar as preocupações, os amparos e as proteções, visulaiza-se um idoso estigmatizado como frágil, limitado, impotente e incapaz de assumir a gestão da própria vida.

As políticas públicas brasileiras envolvendo o idoso, de 1920 até 2003, 
foram analisadas por Correa et al. (2010), o qual sopesou o impacto dessas medidas no processo de envelhecimento. Verificou-se que as legislações dirigidas ao idoso, diante do crescimento do envelhecimento populacional, começaram a surgir, a fim de controlar os rumos do processo de envelhecer, entendendo que, com a nova visão das políticas públicas, tem-se uma imagem do envelhecimento saudável e não mais atrelado à doença.

Scortegagna et al. (2010) identificaram o papel da educação como possibilidade de mudanças para os idosos, analisando a inserção desses indivíduos no contexto educativo. Evidenciou-se que o idoso é visto como adulto nas políticas públicas que se referem à educação, desaparecendo suas peculiaridades, as quais são evidenciadas nas legislações para terceira idade, concluíndo que se deve, portanto, pensar na estruturação das políticas públicas, para garantir direitos para esses sujeitos.

As condições de letramento de um grupo de idosos frequentadores de uma Unidade de Saúde de Curitiba-PR foram analisadas por Massi et al. (2010). Aplicou-se um questionário com dados socio-demográfico-culturais e um texto para localizar informações explícitas e fazer inferências. Pode-se ressaltar que a população idosa tem acesso restrito à linguagem escrita, pois apesar de afirmarem ler textos de jornais e revistas, não conseguiram extrair informações simples do cotidiano social. Contudo, os autores concluem que o EI depende de implementação de políticas públicas, para que ocorra a prática do letramento em consonância com o processo de envelhecimento.
De acordo com Souza et al. (2010), realizou-se um levantamento, em documentos oficiais, do tema violência, bem como das diretrizes que orientam a atenção do setor para o cuidado da saúde do idoso na questão do atendimento e também da prevenção. Foram encontradas cinco importantes normativas: a) política nacional do idoso, b) política nacional da saúde da pessoa idosa, c) política nacional de atenção às urgências, d) política nacional de saúde da pessoa portadora de deficiência, e) pacto pela saúde. Além desses documentos, usou-se dois marcos referênciais - o EI e a Política Nacional de Redução de Morbimortalidade por Acidente e Violência (PNRMAV). Os documentos que abordam a questão da violência são: o Pacto pela Saúde, O EI, a PNRMAV, a Política Nacional de Saúde do Idoso e a Política de Promoção da Saúde. Desse modo, concluíram que o tema está aparecendo, de forma gradual, nas políticas públicas, apontando o EI como melhor documento em termos de objetividade e assertividade. Logo, constataram que a PNRMAV não vem sendo aplicada e o tema violência não é abordado como deveria, ou seja, do ponto de vista das manifestações e da frequência que ocorrem.

Outro tema abordado por Cavalcanti et al. (2007) foi a percepção dos gestores e profissionais de saúde em relação à atenção aos idosos vítimas de violência, no município do Rio de Janeiro. A pesquisa foi realizada de forma qualitativa com um total de vinte entrevistados. $\mathrm{Na}$ fase exploratória, para identificar os serviços de atendimento que atendem idosos vítimas de violência, realizou-se 
cinco entrevistas com gestores. As questões aos entrevistados (médicos, gestores coordenadores, psiquiatras, fisioterapeutas e assistentes sociais) versavam a respeito do roteiro de entrevistas e do fluxo de atendimento aos idosos vítimas de violência. Alguns profissionais não distinguiram maus tratos de violência. Para evitar a violência, um dos gestores destaca a questão da educação, dizendo que se deve educar a família para a doença. Com os relatos, foi apontada uma desarticulação da delegacia do idoso com o sistema de saúde, concluindo que o idoso não é atendido multidisciplinarmente no que se refere à atenção integral, havendo dificuldades estruturais e organizacionais vivenciadas pelo sistema de saúde.

Outra questão estudada diz respeito ao perfil do agressor e do idoso que sofreu violência em Salvador, em 2008, bem como o tipo de agressão mais frequente. Pinheiro et al. (2011) buscaram, em um estudo observacional e decritivo, registrados na delegacia especial de atendimento ao idoso de Salvador acompanhar 1.999 casos. O estudo mostrou que a maioria das vítimas eram mulheres $(57,1 \%)$, casadas $(32,9 \%)$ e aposentadas $(61,3 \%)$. Os agressores eram, na sua maioria, homens $(60,4 \%)$, solteiros $(65,4 \%)$ e filhos das vítimas $(21,3 \%)$. As agressões mais registradas foram $o$ desrespeito com o EI $(27,3 \%)$ e a ameaça $(26,4 \%)$, concluíndo que as políticas de proteção ao idoso precisam ser divulgadas para, assim, garantir a assistência integral ao idoso.

\section{Considerações finais}

As publicações, desde a promulgação do Estatuto do Idoso, em 2003, detém-se, frequentemente, a investigar a violência contra o idoso, seus tipos, os meios de ocorrer, o perfil do idoso e do agressor (em 29\% dos textos sobre a temática do do EI). Também, identificou-se o importante papel que a educação cumpre para modificar o pensar das gerações futuras, refletindo na diminuição da violência, no respeito e na garantia dos direitos da pessoa idosa.

Nesse contexto, é imprescindível a reflexão científica sobre um instrumento tão importante e garantidor de direitos, para que se consiga encontrar medidas de proteger, ainda mais, essa camada vulnerável da sociedade.

\section{An overview of the first decade after the promulgation of the Brazilian Statute for the Elderly}

\section{Abstract}

This sistematic review focuses on the Brazilian Statute for the Elderly (SE) law $10.741 / 2003$, which regulates the rights of the elderly living in Brazil. Twenty-three articles were identified, from which 21 were included in this literature review. These addressed: the knowledge of elderly, who attended a Open University of the Third Age, regarding citizenship and ageing (one publication); health professional's perception who worked at a Geriatric hospital about the changes in the assistance to the elderly after the promulgation of SE (one publication); different perspectives of violence (types of violence, profiles of the aggressor 
and the assaulted, mandatory notification from health professional about violence (six publications)]; education regarding the improvement of the relationships and respect between the elderly and younger generations and the elderly's education itself (three publications)]; the legislative landscape regarding the elderly's rights (two publications); public policies in Brazil regarding the elderly (four publications); the rights established in the Status of the Elderly (one publication); the presence of a nutritionist in the SE Status (one publication); the description of the experience of health professional about workshop activities concerning leisure and health knowledge of a group of elderly from the Estratégia Saúde da Família (Family Health Strategy) (one publication); the content analysis of the $\mathrm{SE}$, identifying the image and making sense of ageing (one publication). It was observed that the most frequent topic in the literature is the violence against the elderly ( $29 \%$ of the publications about the SE).

Keyword: Aging. Aged. Elderly. Legislation. Publications.

\section{Referências}

BRASIL. Portaria GM/MS 703, de 12 de abril de 2002. Disponível em: http://200.189.113.52/ ftp/saude_idoso/02PortariaGM703.doc. Acesso em: 28 jun. 2011.

BEZERRA, R. M. N. Instituição do Estatuto do Idoso e conceito do idoso. In: PINHEIRO, N. M. (Org.) Estatuto do idoso comentado. 3. ed. São Paulo: Servanda, 2012.

CAMARGOS, C. N.; MENDONÇA, C. A.; VIANA, E. M. B. Política, estado e sociedade: o estatuto do idoso e a atenção à saúde. Comunicação em Ciências da Saúde, Brasília, v. 17, n. 3, p. 217-227, jul./set. 2006.
CAMARGOS, C. N. Panorama de interiores: o profissional nutricionista e o estatuto do idoso. Revista Brasileira de Geriatria e Gerontologia, Rio de Janeiro, v. 12, n. 3, p. 489-496, set./dez. 2009.

CAVALCANTE, P. M. T.; FREITAS, M. C. Em busca de um viver saudável: relato de experiência com um grupo de idosos cadastrados na estratégia saúde da família de Pacatuba (CE). Revista da Rede de Enfermagem do Nordeste, Fortaleza, v. 8, n. 1, p. 92-99, jan./ abr. 2007.

CAVALCANTI, M. L. T.; SOUZA E. R. Percepções de gestores e profissionais de saúde sobre a atenção aos idosos vítimas de violências no município do Rio de Janeiro. Ciência \& Saúde Coletiva, Rio de Janeiro, v. 15, n. 6, p. 2699-2708, set. 2010.

CÍCERO, M.T. Saber envelhecer. Trad. de Paulo Neves. Porto Alegre: LP\&M, 2010.

CORREA, M. R.; FRANÇA, S. A. M.; HASHIMOTO, F. Políticas públicas: a construção de imagens e sentidos para o envelhecimento humano. Estudos Interdisciplinares sobre o Envelhecimento, Porto Alegre, v. 15, n. 2, p. 219-238, dez. 2010.

GIANISELLE, F. G. et al. Estatuto do idoso: aspectos jurídicos, sociais e culturais. Kairós Gerontologia, São Paulo, v. 7, n. 2, p. 127-149, dez. 2004.

GOMES, L.; VIANNA, C. Violência contra o ancião. Jornal Brasileiro de Medicina, Rio de Janeiro, v. 94, n. 1 e 2, p. 26-28, jan./fev. 2008.

HEBLING, E.; RODRIGUES, C. K. O estatuto do idosos e a saúde bucal. Robrac, Goiânia, v. 15, n. 39 , p. 51-56, jun. 2006.

IBGE - INSTITUTO BRASILEIRO DE GEOGRAFIAE ESTATÍSTICA. Censo demográfico 2010. Disponível em: http://www.censo2010. ibge.gov.br/ Acesso em: 2 maio 2011.

JUSTO, J. S.; ROZENDO, A. S. A velhice no estatuto do idoso. Estudos e Pesquisas em Psicologia, Rio de Janeiro, v. 10, n. 2, p. 471-489, ago. 2010. 
LAKS, J.; WERNER, J.; MIRANDA-SÁ JÚNIOR, L. S. Psiquiatria forense e direitos humanos nos polos da vida: criança, adolescente e idosos. Revista Brasileira de Psiquiatria, São Paulo, v. 28, sup. 2, p. 80-85, out. 2006.

LOCH, J. A. et al. Envelhecimento e bioética. In: CASADO, M., LUNA, F. (Orgs.). Cuestiones de bioética en y desde lationoamérica. Barcelona: Civitas, 2012. p. 292-293.

MARTINEZ, N. M. Comentários ao Estatuto do Idoso. São Paulo: LTr, 2012.

MARTINS, M. S.; MASSAROLLO, M. C. K. B. Mudanças na assistência ao idoso após promulgação do estatuto do idoso segundo profissionais de hospital geriátrico. Revista da Escola de Enfermagem da USP, São Paulo, v. 42, n. 1, p. 26-33, mar. 2008.

MASSI, G. et al. Práticas de letramento no processo de envelhecimento. Revista Brasileira de Geriatria e Gerontologia, Rio de Janeiro, v. 13, n. 1, p. 59-72, jan./abr. 2010.

MIYATA, D. F. et al. Políticas e programas na atenção à saúde do idoso: um panorama nacional. Arquivos de Ciências da Saúde da UNIPAR, Umuarama, v. 9, n. 2, p. 135-140, maio 2005.

MOIMAZ, S. A. S. et al. O idoso no Brasil: aspectos legislativos de relevância para profissionais de saúde. Revista Espaço para Saúde, Londrina, v. 10, n. 2, p. 61-69, jun. 2009.

PEREIRA, R. J.; COTTA, R. M. M.; PRIORE, S. E. Políticas sobre envelhecimento e saúde no mundo. Mundo Saúde, São Paulo, v. 29, n. 4, p. 475-483, out. 2005.

PERES, A. P. A. B. Proteção aos idosos. Curitiba: Juruá, 2009.

PINHEIRO, J. S.; SILVA, R. C.; ANDRADE, M. C. Perfil dos idosos que sofreram violência atendidos em uma instituição de Salvador no ano de 2008. Revista Baiana de Saúde Pública, Salvador, v. 35, n. 2, p. 264-276, abr./jun. 2011.
PONTES, P. A. G. Proteção integral. In: PINHEIRO, N. M. (Org.) Estatuto do idoso comentado. 3. ed. São Paulo: Servanda, 2012. p. 47.

SALIBA, O. et al. Responsabilidade do profissional de saúde sobre a notificação de casos de violência doméstica. Revista de Saúde Pública, São Paulo, v. 41, n. 3, p. 472-477, jun. 2007.

SCORTEGAGNA, P. A.; OLIVEIRA, R. C. S. Educação: integração, inserção e reconhecimento social para o idoso. Kairós Gerontologia, São Paulo, v. 13, n. 1, p. 53-72, jun. 2010.

SHAKESPEARE, W. Rei lear. Tradução de Pietro Nassetti. São Paulo: Martins Claret, 2007.

SILVA, A. C. A. P. Conhecimento, cidadania e direito do idoso: relatos pós-lei $\mathrm{n}$. 10.741/2003. Revista Brasileira de Geriatria e Gerontologia, Rio de Janeiro, v. 11, n. 1, p. 45-55, jan.-jun. 2008.

SOUZA, E. R.; MINAYO, M. C. S. Inserção do tema violência contra a pessoa idosa nas políticas públicas de atenção à saúde no Brasil. Ciência \& Saúde Coletiva, Rio de Janeiro, v. 15, n. 6, p. 2659-2668, set. 2010.

WHITAKER, D. C. A. O idoso na contemporaneidade: a necessidade de se educar a sociedade para as exigências desse "novo" ator social, titular de direitos. Cadernos Cedes, Campinas-SP, v. 30, n. 81, p. 179-188, maio/ago. 2010. 